\title{
Geographic Distribution of Trichothecene Chemotypes of the Fusarium graminearum Species Complex in Major Winter Wheat Production Areas of China
}

\author{
Cheng-mei Shen, Institute of Plant Protection, Jiangsu Academy of Agricultural Sciences, Nanjing 210014, China and College of Plant \\ Protection, Nanjing Agricultural University, Nanjing 210095, China; Ying-chun Hu, Hai-yan Sun, and Wei Li, Institute of Plant \\ Protection, Jiangsu Academy of Agricultural Sciences; Jian-hua Guo, College of Plant Protection, Nanjing Agricultural University; and \\ Huai-gu Chen, Institute of Plant Protection, Jiangsu Academy of Agricultural Sciences
}

\begin{abstract}
Shen, C.-M., Hu, Y.-C., Sun, H.-Y., Li, W., Guo, J.-H., and Chen, H.-G. 2012. Geographic distribution of trichothecene chemotypes of the Fusarium graminearum species complex in major winter wheat production areas of China. Plant Dis. 96:1172-1178.

Fusarium head blight, caused by members of the Fusarium graminearum species complex (FGSC), is among the most destructive and economically important diseases of small grain crops, including wheat. To determine the phylogenetic species and mycotoxin (trichothecene) chemotypes of the FGSC in the major winter-wheat-producing areas of China, 530 isolates were collected from diseased wheat during the years 2008, 2009, and 2010, and typed using a polymerase chain reaction-based trichothecene genotype assay. Virulence of isolates with different chemotypes was also compared. Of the 530 isolates typed, 348 were $F$. asiaticum and 182 were $F$. graminearum. Subdividing the 530 isolates by the trichothecene predicted to be expressed, 482 were of the deoxynivalenol (DON) chemotype and 48 were nivalenol (NIV). Acetylated derivatives of DON included 3-acetyldeoxynivalenol (3-

AcDON; 300 isolates), and 15-acetyldeoxynivalenol (15-AcDON; 182 isolates). Chemotypes of the $F$. asiaticum isolates were either 3-AcDON or NIV, with 3-AcDON being predominant. F. graminearum isolates were all of the $15-\mathrm{AcDON}$ chemotype. F. asiaticum was the predominant phylogenetic species in the Yangtze River Basin and $F$. graminearum was dominant in the north of China. Two areas of cooccurrence of trichothecene chemotypes were found. The 3-AcDON and 15-AcDON isolates had similar levels of virulence. The DON isolates were significantly more virulent than those of the NIV. The 3AcDON and 15-AcDON chemotypes were predominant in the Yangtze River Basin and areas north of the Yangtze River Basin, respectively, and it is suggested that geographic distribution is associated with differences in temperature as well as crop rotation systems.
\end{abstract}

Fusarium head blight (FHB), also known as wheat scab, is caused by members of the Fusarium graminearum species complex (FGSC) (15). It is an economically devastating disease worldwide $(48,51)$ and, in China, epidemics of FHB occur frequently in the Yangtze River Basin $(9,51)$. With changes in climate and tillage practices, FHB has gradually spread north from the Yangtze River Basin and now covers more than 10 provinces including Hebei, Henan, Shandong, Anhui, and Jiangsu, which include the major winter-wheat-producing areas in China (9).

The FGSC consists of 16 phylogenetically distinct species (28$30,37,40)$, based on genealogical concordance phylogenetic species recognition of strains characterized morphologically as $F$. graminearum Schwabe (15). Most species appear to be restricted to specific geographic regions (49). In the United States, Europe, and Canada, $F$. graminearum sensu stricto is the principal cause of FHB but this species occurs in other areas of the world as well. In Asia, F. asiaticum predominates but a small population of $F$. graminearum has also been found. In China, $F$. graminearum is primarily found in cooler wheat-growing regions and $F$. asiaticum in the warmer regions $(33,34)$.

Fungal infestation by the FGSC reduces crop yield in terms of disease in the field and grain in storage; grain quality suffers as a result of sterile florets and withered kernels. In addition to the detrimental effects of the FGSC as plant pathogens, contamination of grain by the trichothecene mycotoxins they produce also poses a significant problem. These toxins cause health problems (mycotoxicoses) in humans and other animals by inhibiting ribosomal

Corresponding authors: H.-G. Chen, E-mail: huaigu@ jaas.ac.cn and J.-H. Guo, E-mail: jhguo@njau.edu.cn

Accepted for publication 2 March 2012.

http://dx.doi.org/10.1094/PDIS-11-11-0974-RE

(C) 2012 The American Phytopathological Society protein synthesis $(11,45)$. Both dermal contact and ingestion cause rapid irritation to skin and intestinal mucosa, respectively, leading to dermatitis, emesis, oral lesions, hemorrhaging, systemic immunosuppression, and even cancers $(6,36,39)$.

Trichothecenes are toxic sesquiterpene epoxides produced as secondary metabolites by species of the fungal genera Fusarium, Myrothecium, Stachybotrys, and others $(5,39)$. More than 80 trichothecenes occur naturally, all having a 12,13-epoxytrichothec-9ene ring in common (16) and an olefinic bond with various side chain substitutions (5). They have been classified into four types (A to D), according to their functional groups. Type-B trichothecenes, with a ketone at C-8 (22), are the principal toxins produced by the FGSC, most notably deoxynivalenol (DON, also known as vomitoxin) and nivalenol in wheat. FGSC strains may be classified into three strain-specific chemotypes according to the sets of trichothecene mycotoxin metabolites (8-ketotrichothecenes) they express: nivalenol and its acetylated derivative, 4-acetylnivalenol (NIV), and two acetylated derivatives of DON, 3-acetyldeoxynivalenol (3AcDON), and 15-acetyldeoxynivalenol (15-AcDON) $(26,46)$.

The different toxicological effects of the NIV, 3-AcDON, and $15-A c D O N$ chemotypes make their identification in specific geographic regions important. The DON and NIV chemotypes have been reported in various countries in Africa, Asia, and Europe $(19,26)$. F . graminearum strains with the $15-\mathrm{AcDON}$ chemotype are largely responsible for FHB in North America $(1,2,26)$ although, recently, Gale et al. $(13,14)$ reported a population of $F$. graminearum with 3-AcDON in six Minnesota and North Dakota counties, and discovered NIV populations of $F$. graminearum and F. asiaticum in southern Louisiana. DON and NIV chemotypes have been reported in China $(18,19,29,40)$. However, their exact distribution in the major winter-wheat-producing areas of China is unknown.

Some researchers have found that trichothecenes are also virulence factors in plants $(17,32,39)$. A series of studies indicated that trichothecene was a virulence factor in wheat and enabled the fungus to spread from infected florets into the wheat rachis $(17,31)$. 
It appears that members of the FGSC may differ little in their virulence toward wheat (15). Von der Ohe et al. (42) reported that there was no significant difference in virulence between strains possessing either the 3-AcDON or 15-AcDON chemotype in wheat. However, the chemotypes have been linked to differences in toxicities in animals. DON has been associated with feed refusal (anorexia), vomiting, and suppressed immune functions (10). Some studies have shown that DON and NIV are similar in cytoxicity (12) but, according to others, NIV is much more toxic in humans and domestic animals (27). However, no correlation was found between aggressiveness toward plants and the amount of toxins in the kernels at maturity (3).

Recent technological advances have enabled researchers to survey agricultural areas at risk for FHB by FGSC isolates, to assess the diversity of chemotypes and associate them with species and geographic distribution $(4,7,35)$. Thus, the objectives of the present study were to determine the distribution of the FGSC chemotypes in the major winter-wheat-producing areas of China, to determine the chemotypes of each phylogenetic species and their geographic distribution, and to correlate virulence toward wheat with chemotype.

\section{Material and Methods}

Fusarium strains. Wheat spikes showing typical symptoms of FHB were collected during the years 2008, 2009, and 2010, covering approximately 100 counties from six provinces, including Hebei, Shangdong, Henan, Hubei, Anhui, and Jiangsu. Kernels were rinsed with sterile distilled water and then surface disinfected in $3 \%$ sodium hypochlorite for $3 \mathrm{~min}$. The kernels were then thoroughly rinsed with sterile distilled water and placed on potato dextrose agar (PDA) plates containing streptomycin at $50 \mathrm{mg} / \mathrm{liter}$. Plates were incubated about 4 to 5 days at $25^{\circ} \mathrm{C}$. Colonies were subcultured on new PDA plates, purified using the single-spore method, and, subsequently, stored on PDA slants at $4^{\circ} \mathrm{C}$. In total, 530 isolates were identified as members of the FGSC. Detailed information regarding the isolates is listed in Table 1.

DNA extraction. All isolates were grown on PDA plates at $25^{\circ} \mathrm{C}$ for 5 to 6 days. Mycelia were harvested, frozen in liquid nitrogen, and ground to a fine powder. Total genomic DNA was extracted using the E.Z.N.A. Fungal DNA Mini Kit (Omega Bio-tek, Germany).

Assay for FGSC. The primer pair UBC85F410/UBC85R410 (Table 2) for polymerase chain reaction (PCR) amplification was used to determine whether the isolates belonged to the FGSC (38). Amplification conditions were $94^{\circ} \mathrm{C}$ for $2 \mathrm{~min}$ and 35 cycles of $94^{\circ} \mathrm{C}$ for $1 \mathrm{~min}, 58^{\circ} \mathrm{C}$ for $1 \mathrm{~min}, 72^{\circ} \mathrm{C}$ for $1 \mathrm{~min}$, and $72^{\circ} \mathrm{C}$ for 10 min. PCR amplification was confirmed by electrophoresing $5 \mu \mathrm{l}$ of the PCR reaction mixture into a $1 \%$ agarose gel electrophoresis. When this method failed, a phosphate permease gene sequence was used for phylogenetic analysis to determine whether the isolates were members of the FGSC (28).

Assay for $\boldsymbol{F}$. asiaticum and $\boldsymbol{F}$. graminearum. The primer set H3dStyI/H3R1 (Table 2) was used to amplify a portion (approxi- mately $223 \mathrm{bp}$ ) of the histone H3 gene sequence (41). The amplicon from $F$. asiaticum strains was cleaved into 195 and 28 bp with StyI, while the amplicon from $F$. graminearum strains was cleaved into 191 and 32 bp with EcoRV. The total volume of the reaction mixture was $20 \mu \mathrm{l}$, containing $10 \times$ PCR buffer, $25 \mathrm{mM}$ $\mathrm{MgCl}_{2}, 2.5 \mathrm{mM}$ dNTP, $0.5 \mu \mathrm{M}$ each primer, 5 units of rTaq polymerase (TaKaRa Biotechnology, Dalian, China), and genomic DNA at $5 \mathrm{ng} / \mu \mathrm{l}$. Cyling parameters were $94^{\circ} \mathrm{C}$ for $2 \mathrm{~min} ; 30$ cycles of $94^{\circ} \mathrm{C}$ for $1 \mathrm{~min}, 5^{\circ} \mathrm{C}$ for $1 \mathrm{~min}$, and $72^{\circ} \mathrm{C}$ for $1 \mathrm{~min}$; and $72^{\circ} \mathrm{C}$ for $10 \mathrm{~min}$. The PCR amplicon of $223 \mathrm{bp}$ was confirmed by using $5 \mu \mathrm{l}$ of the PCR mixture for $1 \%$ agarose gel electrophoresis. A 100bp ladder (TaKaRa Biotechnology) was used as a size standard. The remaining $8 \mu \mathrm{l}$ of the PCR mixture was used for digestion, with StyI (Fermentas) or EcoRV (Fermentas) at $10 \mathrm{U} / \mu \mathrm{l}$ in a $20-\mu \mathrm{l}$ reaction mixture, followed by incubation at $37^{\circ} \mathrm{C}$ for $4 \mathrm{~h}$ and then electrophoresis using an $8 \%$ polyacrylamide gel.

Chemotyping and identification of DON and NIV chemotypes. Chemotypes of the FGSC isolates were determined using the specific primer pair described by $\mathrm{Li}$ et al. (24). The primer set ToxP1/P2 (Table 2) derived from the intergenic sequences between the Tri5 and Tri6 genes was synthesized and used for identification of the DON and NIV chemotypes, generating a 300-bp sequence for a DON chemotype and a 360-bp for an NIV chemotype. The amplification reaction used was described by Jennings et al. $(18,19)$.

Identification of 3-AcDON and 15-AcDON chemotypes. Another two primer sets, Tri303F/Tri303R and Tri315F/Tri315R (Table 2), which target the Tri3 gene $(18,19)$, were used to further characterize DON chemotypes of the $F$. graminearum complex as 3- or 15-AcDON. The products were 586 and $864 \mathrm{bp}$, respectively. The amplification reaction used was as described above. The cycling protocol consisted of denaturation at $95^{\circ} \mathrm{C}$ for $4 \mathrm{~min}$; followed by 35 cycles of $94^{\circ} \mathrm{C}$ for $1 \mathrm{~min}, 58^{\circ} \mathrm{C}$ for $50 \mathrm{~s}$, and $72^{\circ} \mathrm{C}$ for $50 \mathrm{~s}$; with a final extension step of $72^{\circ} \mathrm{C}$ for $10 \mathrm{~min}$. PCR products were analyzed as described above.

Virulence assay. In total, 288 isolates (153 15-AcDON, 116 3AcDON, and 19 NIV) that were all isolates collected from six

Table 2. Primer sequences used in this study

\begin{tabular}{llc}
\hline Primer & \multicolumn{1}{c}{ Nucleotide sequence $\left(\mathbf{5}^{\prime} \text { to } \mathbf{3}^{\mathbf{\prime}}\right)^{\mathbf{a}}$} & $\mathbf{C i t e}^{\mathbf{b}}$ \\
\hline ToxP1 & GCCGTGGGGRTAAAAGTCAAA & 24 \\
ToxP2 & TGACAAGTCCGGTCGCACTAGGA & 24 \\
Tri303F & GATGGCCGCAAGTGGA & 19 \\
Tri303R & GCCGGACTGCCCTATTG & 19 \\
Tri315F & CTCGCTGAAGTTGGACGTAA & 19 \\
Tri315R & GTCTATGCTCTCAACGGACAAC & 19 \\
H3dStyI & AGCATCACCYGAACATCGCATCATCCCATG & 30 \\
H3R1 & TTGGACTGGATRGTAACACGC & 30 \\
UBC85F410 & GCAGGGTTTGAATCCGAGAC & 38 \\
UBC85R410 & AGAATGGAGCTACCAACGGC & 38 \\
\hline
\end{tabular}

${ }^{\mathrm{a}} \mathrm{R}=\mathrm{A}+\mathrm{G}$ and $\mathrm{Y}=\mathrm{C}+\mathrm{T}$.

${ }^{\mathrm{b}}$ Reference from literature cited.

Table 1. Trichothecene chemotypes and phylogenetic species of Fusarium isolates collected in six provinces of China

\begin{tabular}{|c|c|c|c|c|c|c|}
\hline \multirow[b]{2}{*}{ Area } & \multirow[b]{2}{*}{ Isolates } & \multicolumn{2}{|c|}{ Phylogenetic species and number of isolates } & \multicolumn{3}{|c|}{ Chemotype, numbers of isolates ${ }^{a}$} \\
\hline & & Name & Number & 3-AcDON & 15-AcDON & NIV \\
\hline \multirow[t]{2}{*}{ Jiangsu } & 292 & Fusarium graminearum & 16 & 0 & 16 & 0 \\
\hline & & F. asiaticum & 276 & 247 & 0 & 29 \\
\hline \multirow[t]{2}{*}{ Anhui } & 71 & F. graminearum & 15 & 0 & 15 & 0 \\
\hline & & F. asiaticum & 56 & 42 & 0 & 14 \\
\hline \multirow[t]{2}{*}{ Henan } & 88 & F. graminearum & 79 & 0 & 79 & 0 \\
\hline & & F. asiaticum & 9 & 7 & 0 & 2 \\
\hline \multirow[t]{2}{*}{ Hebei } & 23 & F. graminearum & 23 & 0 & 23 & 0 \\
\hline & . & F. asiaticum & 0 & 0 & 0 & 0 \\
\hline \multirow[t]{2}{*}{ Shandong } & 31 & F. graminearum & 26 & 0 & 26 & 0 \\
\hline & $\ldots$ & F. asiaticum & 5 & 4 & 0 & 1 \\
\hline \multirow[t]{2}{*}{ Hubei } & 25 & F. graminearum & 23 & 0 & 23 & 0 \\
\hline & $\ldots$ & F. asiaticum & 2 & 0 & 0 & 2 \\
\hline
\end{tabular}

a 3-AcDON = 3-acetyldeoxynivalenol, 15-AcDON = 15-acetyldeoxynivalenol, and NIV = nivalenol. 
provinces in 2009 and 2010 were assayed for virulence toward 'Yangmai158', a partially resistant winter wheat cultivar. In late October, wheat seed were sown in the nursery under open and unprotected conditions, and spaced to avoid cross-contamination. Conidia were prepared using $6 \%$ mung bean both (41). Spore concentration was determined using a hemocytometer and diluted to obtain 1 to $2 \times 10^{5}$ spores $/ \mathrm{ml}$ for inoculation at flower anthesis $(44,50)$. Flowering heads of wheat were point inoculated with the above conidial suspension. The number of spikelets with disease symptoms was counted at $7,14,21$, and 28 days post inoculation (dpi). Virulence was estimated by the mean number of spikelets with disease symptoms. Each isolate was used to inoculate 10 heads for analysis. Statistical analysis was performed using SAS release 6.12 .

\section{Results}

Trichothecene chemotypes of the $F$. graminearum complex. Although several extra bands were also usually amplified, the 332bp amplicon was observed in all 530 isolates, indicating that they were all members of the FGSC. PCR assays with the primer pair ToxP1/P2 revealed that $482(91 \%)$ of the 530 FGSC isolates produced a 300-bp fragment, indicating that all were DON. For the remaining $48(9 \%)$ isolates, a 360-bp PCR product was obtained under the same conditions and, thus, they were classified as NIV (Fig. 1A). To predict which DON derivatives might be produced, all isolates were subjected to PCR assays with two pairs of primers, Tri303F/Tri303R and Tri315F/Tri315R. The results showed that, with the primer pair Tri303F/R, $300(62 \%)$ of the $482 \mathrm{DON}$ chemotypes yielded a 3-AcDON-specific fragment of $586 \mathrm{bp}$ (Fig. 1B). An 864-bp 15-AcDON-specific fragment was amplified from 182 (38\%) DON chemotype isolates with the primer pair Tri315F/R (Fig. 1C). No PCR products were observed from the NIV chemotypes (Fig. 1B and C).
Furthermore, PCR amplicons from 182 isolates using the primer set $\mathrm{H} 3 \mathrm{dStyl} / \mathrm{H} 3 \mathrm{R} 1$ were cleaved by restriction enzyme EcoRV and, therefore, they were identified as $F$. graminearum (Table 1 ). In all, 348 isolates were identified as $F$. asiaticum, because the amplicons were cleaved by the restriction enzyme StyI (Table 1). In the present study, F. asiaticum was represented by $300(86 \%) 3$-AcDON and $48(14 \%)$ NIV isolates. Isolates of the 182 isolates determined to be $F$. graminearum were all 15-AcDON (Table 1).

Geographic distribution of trichothecene chemotypes. Isolates from Hebei, Shandong, Henan, Hubei, Anhui, and Jiangsu province were analyzed for trichothecene chemotype. A map displaying the frequency distribution of the different chemotypes in the six provinces where the Fusarium isolates were collected is show in Figure 2; the chemotypes are indicated. Frequency of the $15-A c D O N$ chemotype was highest in the north of China and decreased from north to south. The NIV and 3-AcDON chemotypes were found mostly in the warmer regions of the Yangtze River Basin in the south, with 3-AcDON showing a decreasing trend from south to north.

Similarly, we determined that the distribution of $F$. asiaticum and $F$. graminearum differed throughout the six provinces. $F$. asiaticum was mainly in the southern regions, along the Yangtze River, and $F$. graminearum was mainly distributed in Hebei, Henan, and Shandong provinces in the north. From south to north, the distribution of $F$. graminearum increased and that of $F$. asiaticum decreased gradually. We also found two regions in which two different chemotypes co-occurred (Fig. 2). In zone A, 3-AcDON and $15-A c D O N$ were sympatric while NIV chemotype was absent. In zone B, 3-AcDON and NIV were found.

Virulence of FGSC isolates during wheat infection in the field. The results indicated that variation in virulence appeared to be an isolate-specific rather than species-specific characteristic. The mean number of symptomatic spikelets was significantly
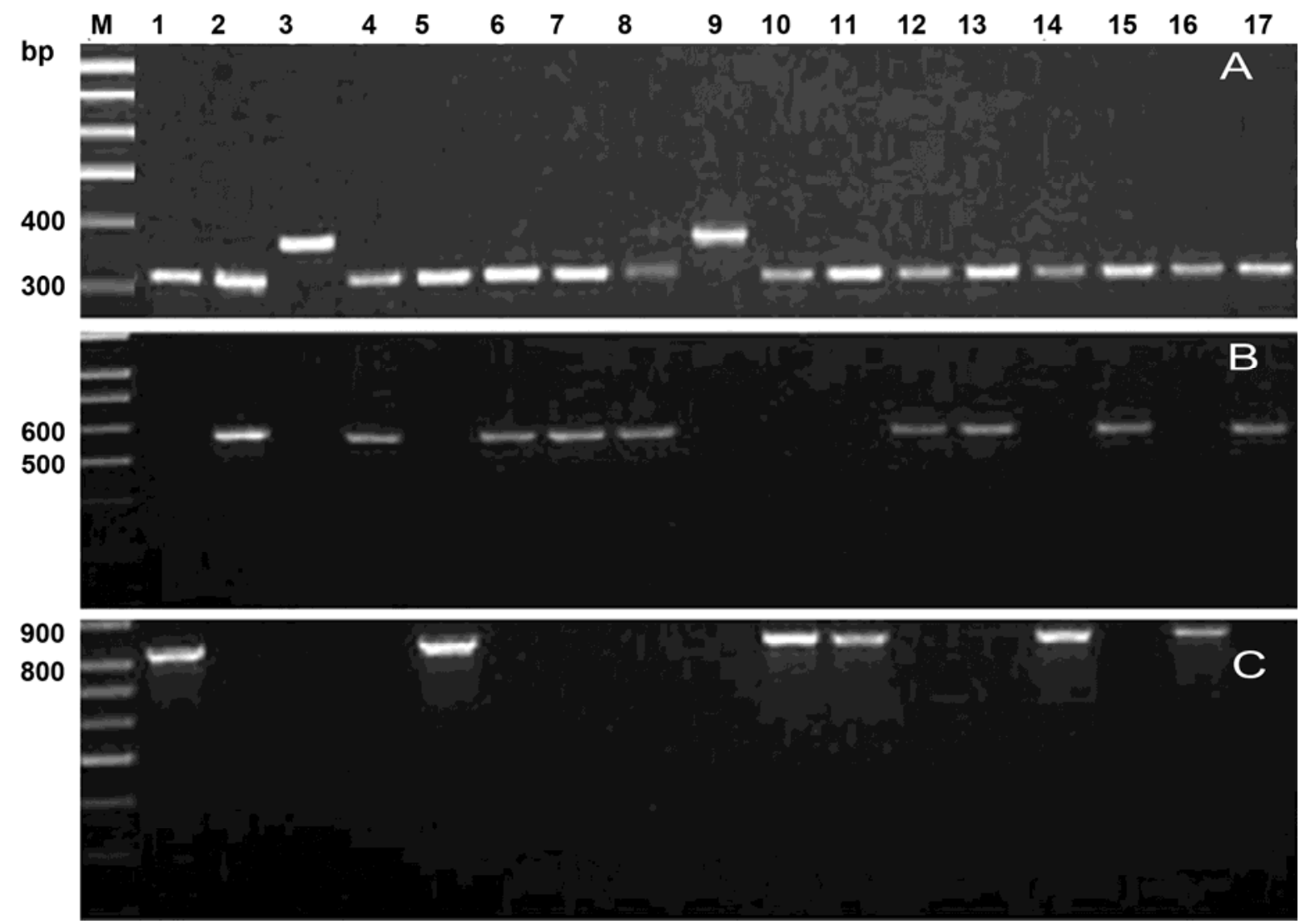

Fig. 1. Polymerase chain reaction-based prediction of Fusarium graminearum species complex chemotype. A, Deoxynivalenol (300 bp) and nivalenol (360 bp), primer set ToxP1/P2; B, 3-acetyldeoxynivalenol (586 bp), primer set Tri303F/R; and C, 15-acetyldeoxynivalenol (864bp), primer set Tri315F/R. M: 100-bp ladder. Lanes 1-8: F09236, F0985, F09204, F0990, F09242, F0991, F0992, F0993, F09260, F09243, F09246, F0994, F0995, F09247, F0987, F09248, F0988, F0989. 
different $(P=0.0013)$ among different chemotypes. There was no obvious difference in virulence among isolates of the 3-AcDON and 15-AcDON chemotypes at 7, 14, 21, or 28 dpi (Fig. 3A and D). However, there were significant differences $(P=0.0001)$ between the DON and NIV chemotypes at 7, 14, 21, and 28 dpi (Fig. 3B and E). We also compared virulence of 149 isolates of $F$. graminearum with 139 isolates of $F$. asiaticum. The results showed that there was no significant difference $(P=0.80)$ between these phylogenetic species on the observed days after inoculation (Fig. 3C and F).

\section{Discussion}

The survey conducted in the present study developed an overall profile of the FGSC in the major winter-wheat-producing regions of China. All three trichothecene chemotypes (3-AcDON, 15-AcDON, and NIV) were found but their distribution differed among the six provinces. Furthermore, the chemotype composition between isolates of $F$. asiaticum and $F$. graminearum was shown to be significantly different. F. asiaticum isolates were represented by 3 -AcDON and NIV chemotypes, while all of the $F$. graminearum isolates were $15-\mathrm{AcDON}$.

In Japan, chemotypes of $F$. graminearum isolates collected from wheat and barley were 3-AcDON and 15-AcDON, while those of F. asiaticum included all three chemotypes (41). Jeon et al. (20) reported that isolates of $F$. asiaticum from Korean maize mainly produced NIV, and $F$. graminearum only DON. The composition of chemotypes appeared to be phylogenetic species dependent (51).
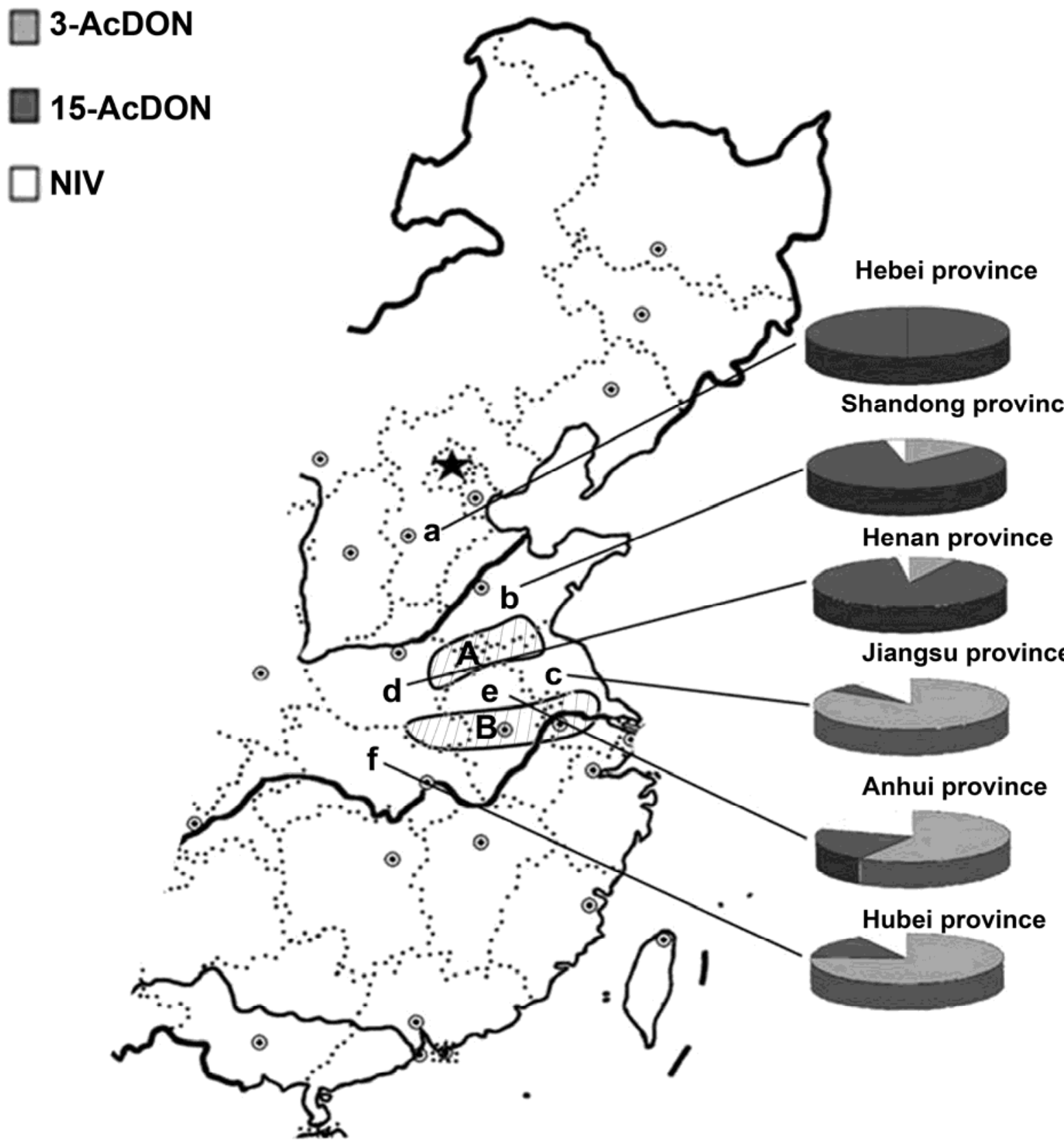

Fig. 2. Trichothecene chemotype frequencies in six provinces of China, based on analyses of 530 Fusarium graminearum species complex isolates. a, Hebei province, with the frequency of 3-acetyldeoxynivalenol (3-AcDON), 15-acetyldeoxynivalenol (15-AcDON), and nivalenol (NIV) being 0, 100, and 0\%, respectively. b, Shandong province, with the frequency of 3-AcDON, 15-AcDON, and NIV being 12.9, 83.9, and 3.2\%, respectively. c, Jiangsu province, with the frequency of 3-AcDON, 15-AcDON, and NIV being $84.6,5.5$, and $9.9 \%$, respectively. d, Henan province, with the frequency of 3-AcDON, 15-AcDON, and NIV being 8.0, 90.0, and 2.0\%, respectively. e, Anhui province, with the frequency of 3-ACDON, 15-ACDON, and NIV being 59.1, 21.1, and 19.8, respectively. f, Hubei province, with the frequency of 3-AcDON, 15-AcDON, and NIV being 76,16 , and $8 \%$, respectively. Area A represents an area of co-occurrence of 3-AcDON and 15-AcDON in the cities of Linyi and Zaozhuang in Shandong province; Shangqiu, Zhoukou, and Zhumadian in Henan province; Bozhou in Anhui province; and Suqian and Lianyungang in Jiangsu province. Area B represents an area of co-occurrence of $3-$ AcDON and NIV in the city of Xinyang in Henan province; Liuan and Haozhou in Anhui province; and Yangzhou, Yancheng, Changzhou, and Zhenjiang in Jiangsu province. 
For Korean isolates collected from rice, F. asiaticum produced NIV and 3-AcDON, whereas $F$. graminearum was represented by 3AcDON and 15-AcDON chemotypes (23). Ward et al. (47) also reported that, in North America, F. graminearum isolates included 3-AcDON and 15-AcDON chemotypes. All of the above results were slightly different from those of the present study. We conclude that the prevalence of trichothecene chemotypes may be correlated with phylogenetic species, host, and geographic origin.

In this study, we found that $F$. graminearum was mainly distributed in the north of China, while $F$. asiaticum was mainly in the south. Qu et al. (34) also reported that $F$. graminearum was mainly in the cooler regions of China, where the annual average temperature was $15^{\circ} \mathrm{C}$ or lower. In contrast, the vast majority of $F$. asiaticum isolates were collected from warmer regions, where the annual average temperature was above $15^{\circ} \mathrm{C}$ and where FHB epidemics occurred frequently. Analysis of Japanese isolates also revealed the presence and differential distribution of $F$. graminearum and $F$. asiaticum in Japan: $F$. graminearum was found predominantly in the north, especially in the Hokkaido area, while $F$. asiaticum was more frequently found in southern regions (41).

Differences in population percentages of $F$. graminearum and $F$. asiaticum (which also differ in their chemotype composition) may also be explained in terms of differences in the geographic distribution of the 3-AcDON, 15-AcDON, and NIV chemotypes. The 3AcDON and NIV chemotypes were mainly in the southern warmer regions, whereas $15-\mathrm{AcDON}$ was more prevalent in the cooler regions of the north (Fig. 2). Similar results were reported previously by Zhang et al. (51) and Ji et al. (21). The trichothecene chemotype distribution shown in the present study was also correlated with temperature patterns. Nevertheless, we found that the distribution of chemotypes was not fully associated with latitude. Xinyang in Henan and Yangzhou in Jiangsu were at approximately the same latitude but only $15-A c D O N$ was found in Xinyang and only 3-AcDON and NIV in Yangzhou. It may be that these differences were due to other factors such as relative humidity and crop rotation practices.

We also found two zones in the six provinces where at least two chemotypes, 3-AcDON and 15-AcDON or 3-AcDON and NIV, coexisted. 3-AcDON and 15-AcDON were found in zone A (Fig. 2) along the Huai River, an area that includes Suqian city in Jiangsu province, Fuyang city in Anhui province, and Zhumadian city in Henan province. In zone B (Fig. 2) 3-AcDON and NIV coexisted but no isolates of the 15-AcDON chemotype were found. Besides the obvious temperature difference, different crop rotation systems were employed in the two zones. Zone A transitions between rice-wheat and maize-wheat plantings, although maizewheat is more frequent. In zone B, the main crop over the summer is rice. However, the distance between Zhumadian and Xinyang in Henan is just $100 \mathrm{~km}$. The climates of these cities are similar but they belong to zone A and zone B, respectively. Therefore, in addition to temperature, the distribution of chemotypes may also be influenced by the crops planted.

Carter et al. (8) reported that virulence of what we now recognize as FGSC was associated with the genetic groups of the isolates. Goswami et al. (15) found that the amount of mycotoxin produced had an effect on virulence but not the chemotype itself, and variation in virulence was strain specific rather than species specific. In this study, we found that isolates with different chemotypes had significant differences in virulence. Although there was no difference between the 3-AcDON and 15-AcDON chemotypes
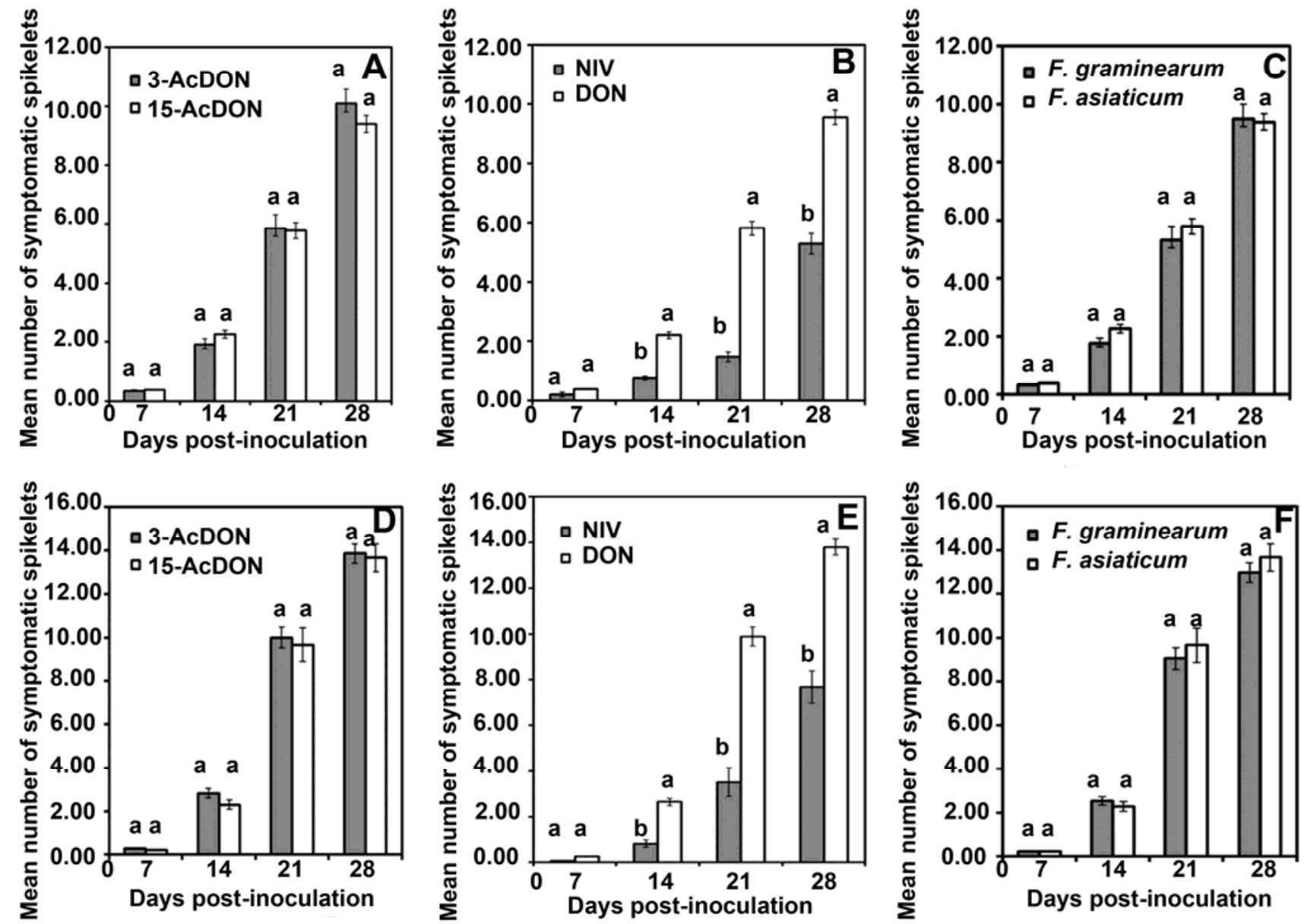

Fig. 3. Virulence of isolates representing different chemotypes. A-C, Virulence results for Fusarium graminearum species complex isolates of different chemotypes in the year 2009; D-F, results for the year 2010. Lowercase "a" and "b" refer to deviation. The same letter within a column indicates no significantly different as determined by SAS. 3-AcDON = 3-acetyldeoxynivalenol, 15-AcDON = 15-acetyldeoxynivalenol, NIV = nivalenol, and DON = deoxynivalenol. 
in virulence (Fig. 3A), there were differences between DON and NIV at 7, 14, 21, and 28 dpi (Fig. 3B). Virulence of the DON isolates was significantly higher than that of the NIV. We also determined that there was no significant difference in virulence between isolates of $F$. graminearum and $F$. asiaticum. This is the first report regarding relatedness between chemotypes and their virulence based on large numbers of isolates.

DON concentration in the kernels at maturity was also analyzed by high-performance liquid chromatography. The 3-AcDON and 15 -AcDON isolates produced similar amounts of mycotoxin. However, mycotoxin production by the DON chemotype isolates was significantly higher than NIV (data not shown). Previous studies disagree over the connection between trichothecene accumulation and virulence $(15,25,43)$. Ward et al. (47) concluded that significantly higher DON accumulation was not correlated with higher virulence. Von der Ohe et al. (42) also determined that the average DON production by $3-A c D O N$ strains was significantly higher than for $15-A c D O N$, whereas both were similarly virulent on wheat. These results need to be verified further. Our results provide a foundation for further determination and monitoring of Fusarium type $\mathrm{B}$ trichothecene mycotoxins at both the regional and national levels. We are continuing to analyze the correlation between virulence and mycotoxin production.

\section{Acknowledgments}

This research was funded by the National Non-Profit Industry-Funded Research and Special Projects (nyhyzx3-15) and by funds earmarked for the China Agricultural Research System (nycytx-03). We thank all of the technicians and students who helped in conducting the field trials and disease evaluations at each location, and Medjaden Bioscience Limited for assisting in preparation of this manuscript.

\section{Literature Cited}

1. Abbas, H. K., Mirocha, C. J., and Tuite, J. 1986. Natural occurrence of deoxynivalenol, 15-acetyl-deoxynivalenol, and zearalenone in refusal factor corn stored since 1972. Appl. Environ. Microbiol. 51:841-843.

2. Abramson, D., Clear, R. M., and Smith, D. M., 1993. Trichothecene production by Fusarium spp. isolated from Manitoba grain. Can. J. Plant Pathol. 15:147-152.

3. Alvarez, C. L., Somma, S., Moretti, A., and Pinto, V. F. 2010. Aggressiveness of Fusarium graminearum sensu stricto isolates in wheat kernels in Argentina. J. Phytopathol. 158:173-181.

4. Astolfi, P., dos Santos, J., Schneider, L., Gomes, L. B., Silva, C. N., Tessmann, D. J., and Del Ponte, E. M. 2011. Molecular survey of trichothecene genotypes of Fusarium graminearum species complex from barley in Southern Brazil. Int. J. Food Microbiol. 148:197-201.

5. Bennett, J. W., and Klich, M. 2003. Mycotoxins. Clin. Microbiol. Rev. 16:497-516.

6. Bimczok, D., Döll, S., Rau, H., Goyarts, T., Wundrack, N., Naumann, M., Dänicke, S., and Rothkötter, H. J. 2007. The Fusarium toxin deoxynivalenol disrupts phenotype and function of monocytederived dendritic cells in vivo and in vitro. Immunobiology 212:655-666.

7. Boutigny, A. L., Ward, T. J., Van Coller, G. J., Flett, B., Lamprecht, S. C., O'Donnell, K., Viljoen, A., and USDA, ARS. 2011. Analysis of the Fusarium graminearum species complex from wheat, barley and maize in South Africa provides evidence of species-specific differences in host preference. Fungal Genet. Biol. 48:914-920.

8. Carter, J. P., Rezanoor, H. N., Holden, D., Desjardins, A. E., Plattner, R. D., and Nicholson, P. 2002. Variation in pathogenicity associated with the genetic diversity of Fusarium graminearum. Eur. J. Plant Pathol. 108:573583 .

9. Chen, L. F., Bai, G. H., and Desjardins, A. E., 2000. Recent advances in wheat head scab research in China. Pages 258-273 in: Proc. Int. Symp. Wheat Improvement for Scab Resistance. Suzhou and Nanjing, China.

10. Desjardins, A. E. 2006. Fusarium Mycotoxins: Chemistry, Genetics, and Biology. American Phytopathological Society, St. Paul, MN.

11. Desjardins, A. E., and Proctor, R. H. 2007. Molecular biology of Fusarium myctotoxins. Int. J. Food Microbiol. 119:47-50.

12. Eriksen, G. S., Pettersson, H., and Lundh, T. 2004. Comparative cytotoxicity of deoxynivalenol, nivalenol, their acetylated derivatives and de-epoxy metabolites. Food Chem. Toxicol. 42:619-624.

13. Gale, L. R., Harrison, S. A., Ward, T. J., O’Donnell, K., Milus, E. A., Gale, S. W., and Kistler, H. C. 2010. Nivalenol-type population of Fusarium graminearum and $F$. asiaticum are prevalent on wheat in southern Louisiana. Phytopathology 101:124-134.

14. Gale, L. R., Ward, T. J., Balmas, V., and Kistler, H. C. 2007. Population subdivision of Fusarium graminearum sensu stricto in the Upper Midwestern United States. Phytopathology 97:1434-1439.

15. Goswami, R. S., and Kistler, H. C. 2005. Pathogenicity and in planta myco- toxin accumulation among members of the Fusarium graminearum species complex on wheat and rice. Phytopathology 95:1397-1404.

16. Hsueh, C. C., Liu, Y., and Freund, M. S. 1999. Indirect electrochemical detection of type-B trichothecene mycotoxins. Anal. Chem. 71:4075-4080.

17. Jansen, C., von Wettstein, D., Schāfer, W., Kogel, K. H., Felk, A., and Maier, F. K. 2005. Infection patterns in barley and wheat spikes inoculated with wild type and trichodiene synthase gene disrupted Fusarium graminearum. Proc. Natl. Acad. Sci. USA 102:16892-16897.

18. Jenning, P., Coates, M. E., Turner, J. A., Chandler, E. A., and Nicholson, P. 2004. Determination of deoxynivalenol and nivalenol chemotypes of Fusarium culmorum isolates from England and Wales by PCR assay. Plant Pathol. 53:182-190.

19. Jennings, P., Coates, M. E., Walsh, K., Turner, J. A., and Nicholson, P. 2004. Determination of deoxynivalenol- and nivalenol-producing chemotypes of Fusarium graminearum isolated from wheat crops in England and Wales. Plant Pathol. 53:643-652.

20. Jeon, J. J., Kim, H., Kim, H. S., Zeller, K. A., Lee, T., Yun, S. H., Bowden, R. L., Leslie, J. F., and Lee, Y. W. 2003. Genetic diversity of Fusarium graminearum from maize in Korea. Fungal Genet. Newsl. 50 (Suppl.):438.

21. Ji, L., Cao, K., Hu, T., and Wang, S. 2007. Determination of deoxynivalenol and nivalenol chemotypes of Fusarium graminearum isolates from China by PCR assay. J. Phytopathol. 155:505-512.

22. Krska, R., Baumgartner, S., and Josephs, R. 2001. The state-of-the-art in the analysis of type-A and -B trichothecene mycotoxins in cereals. Fresen. J. Anal. Chem. 371:285-299.

23. Lee, J., Chang, I. Y., Kim, H., Yun, S. H., Leslie, J. F., and Lee, Y. W. 2009 Genetic diversity and fitness of Fusarium graminearum populations from rice in Korea. Appl. Environ. Microbiol. 75:3289-3295.

24. Li, H. P., Wu, A. B., Zhao, C. S., Scholten, O., Löffler, H., and Liao, Y. C. 2005. Development of a generic PCR detection of deoxynivalenol and nivalenol chemotypes of Fusarium graminearum. FEMS Microbiol. Lett. 243:505-511.

25. Miedaner, T., Schilling, A. G., and Geiger, H. H., 2004. Competition effects among isolates of Fusarium culmorum differing in aggressiveness and mycotoxin production on heads of winter rye. Eur. J. Plant Pathol. 110:63-70.

26. Miller, J. D., Greenhalgh, R., Wang, Y. Z., and Lu, M. 1991. Trichothecene chemotypes of three Fusarium species. Mycologia 83:121-130.

27. Minervini, F., Fornelli, F., and Flynn, F. M., 2004. Toxicity and apoptosis induced by the mycotoxins nivalenol, deoxynivalenol and fumonisin B1 in a human erythroleukemia cell line. Toxicol. In Vitro 18:21-28.

28. O'Donnell K., Kistler, H. C., Tacke, B. K., and Casper, H. H. 2000. Gene genealogies reveal global phylogeographic structure and reproductive isolation among lineages of Fusarium graminearum, the fungus causing wheat scab. Proc. Natl. Acad. Sci. USA 97:7905-7910.

29. O’Donnell K., Ward, T. J., Aberra, D., Kistler, H. C., Aoki, T., Orwig, N., Kimura, M., Bjornstad, A., and Klemsdal, S. S. 2008. Multilocus genotyping and molecular phylogenetics resolve a novel head blight pathogen within the Fusarium graminearum species complex from Ethiopia. Fungal Genet. Biol. 45:1514-1522.

30. O'Donnell, K., Ward, T. J., Geiser, D. M., Kistler, H. C., and Aoki, T. 2004 Genealogical concordance between the mating type locus and seven other nuclear genes supports formal recognition of nine phylogenetically distinct species within the Fusarium graminearum clade. Fungal Genet. Biol. 41:600-623.

31. Proctor, R. H., Hohn, T. M., and McCormick, S. P., 1995. Reduced virulence of Gibberella zeae caused by disruption of a trichothecene toxin biosynthetic gene. Mol. Plant-Microbe Interact. 8:593-601.

32. Proctor, R. H., Hohn, T. M., and McCormick, S. P. 1997. Restoration of wild type virulence to Tri5 disruption mutants of Gibberella zeae via gene reversion and mutant complementation. Microbiology 143:2583-2591.

33. Qu, B., Li, H. P., Zhang, J. B., Huang, T., Carter, J., Liao, Y. C., and Nicholson, P. 2008. Comparison of genetic diversity and pathogenicity of Fusarium head blight pathogens from China and Europe by SSCP and seedling assays on wheat. Plant Pathol. 57:642-651.

34. Qu, B., Li, H. P., Zhang, J. B., Xu, Y. B., Huang, T., Wu, A. B., Zhao, C. S Carter, J., Nicholson, P., and Liao, Y. C. 2008. Geographic distribution and genetic diversity of Fusarium graminearum and F. asiaticum on wheat spikes throughout China. Plant Pathol. 57:15-24.

35. Reynoso, M. M., Ramirez, M. L., Torres, A. M., and Chulze, S. N. 2011 Trichothecene genotypes and chemotypes in Fusarium graminearum strains isolated from wheat in Argentina. Int. J. Food Microbiol. 145:444-448.

36. Sakai, A., Suzuki, C., Masui, Y., Kuramashi, A., Takatori, K., and Tanaka, N. 2007. Activities of mycotoxins derived from Fusarium and related substances in a short-term transformation assay using v-Ha-ras-transfected BALB/3T3 cells (Bhas 42 cells). Mutat. Res. 630:103-111.

37. Sarver, B. A. J., Ward, T. J., Gale, L. R., Karen, B., Kistler, H. C., Takayuki, A., Nicholson, P., Cater, J., and O'Donnell, K. 2011. Novel Fusarium head blight pathogens from Nepal and Louisiana revealed by multilocus genealogical concordance. Fungal Genet. Biol. 48:1096-1107.

38. Schilling, A. G., Moller, E. M., and Geiger, H. H. 1996. Polymerase chain reaction-based assays for species-specific detection of Fusarium culmorum, F. graminearum, and F. avenaceum. Phytopathology 86:515-522.

39. Seong, K. Y., Pasquali, M., Zhou, X. Y., Song, J., Hilburn, K., Cormick, S. M., Dong, Y., Xu, J. R., and Kistler, H. C. 2009. Global gene regulation by 
Fusarium transcription factors Tri6 and Tri10 reveals adaptations for toxin biosynthesis. Mol. Microbiol. 72:354-367.

40. Starkey, D. E., Ward, T. J., Aoki, T., Gale, L. H., Kistler, H. C., Geiser, D. M., Suga, H., Tóth, B., Varga, J., and O’Donnell, K. 2007. Global molecular surveillance reveals novel Fusarium head blight species and trichothecene toxin diversity. Fungal Genet. Biol. 44:1191-1204.

41. Suga, H., Karugia, G. W., Ward, T., Gale, L. R., Tomimura, T., Nakajima, T., Miyasaka, A., Koizumi, S., Kageyama, K., and Hyakumachi, M. 2008. Molecular characterization of the Fusarium graminearum species complex in Japan. Phytopathology 98:159-166.

42. Von der Ohe, C., Gauthier, V., Tamburic-Ilincic, L., Brule-Babel, A., Fernando, W. G. D., Clear, R., Ward, T. J., and Miedaner, T. 2010. A comparison of aggressiveness and deoxynivalenol production between Canadian Fusarium graminearum isolates with 3-acetyl and 15-acetyldeoxynivalenol chemotypes in field-grown spring wheat. Eur. J. Plant Pathol. 127:407-417.

43. Walker, S. L., Leath, S., Hagler, W. M., Jr., and Murphy, J. P. 2001. Variation among isolates of Fusarium graminearum associated with Fusarium head blight in North Carolina. Plant Dis. 85:404-410.

44. Wang, Y. Z., Yang, X. N., and Xiao, Q. P., 1982. The improvement of identification technique of scab (Gibberella zeae Petch) resistance of wheat and the development of resistant sources. Sci. Agric. Sin. 5:67-77.

45. Wannemacher, R. W., Jr., and Wiener, S. L. 1997. Trichothecene mycotoxins. Pages 655-676 in: Medical Aspects of Chemical and Biological War- fare. F. R. Sidell, E. T. Takafuji, and D. R. Franz, eds. Office of the Surgeon General, United States Army, Falls Church, VA.

46. Ward, T. J., Bielawski, J. P., Kistler, H. C., Sullivan, E., and O’Donnell, K. 2002. Ancestral polymorphism and adaptive evolution in the trichothecene mycotoxin gene cluster of phytopathogenic Fusarium. Proc. Natl. Acad. Sci. USA 99:9278-9283.

47. Ward, T. J., Clear, R. M., Rooney, A. P., O'Donnell, K., Gaba, D., Patrick, S., Starkey, D. E., Gilbert, J., Geiser, D. M., and Nowichi, T. W. 2008. An adaptive evolutionary shift in Fusarium head blight pathogen populations is driving the rapid spread of more toxigenic Fusarium graminearum in North America. Fungal Genet. Biol. 25:473-484.

48. Windels, C. E., 2000. Economic and social impacts of Fusarium head blight: changing farms and rural communities in the Northern Great Plains. Phytopathology 90:17-21.

49. Xu, X. M., and Nicholson, P. 2009. Community ecology of fungal pathogens causing wheat head blight. Annu. Rev. Phytopathol. 47:83-103.

50. Yu, G., Chen, L. F., Xie, W. P., and Chai, Y. Q. 2001. Correlation of trichothecene producing potential to pathogenicity of Fusarium graminearum on wheat. J. Nanjing Agric. Univ. 24:19-23.

51. Zhang, J. B., Li, H. P., Dang, F. J., Qu, B., Xu, Y. B., Zhao, C. S., and Liao, Y. C. 2007. Determination of the trichothecene mycotoxin chemotypes and associated geographical distribution and phylogenetic species of the Fusarium graminearum clade from China. Mycol. Res.10:967-975. 